\title{
Temperatura efectiva en estrellas de tipo solar
}

\author{
D. Cornejo ${ }^{1}$, I. Ramírez ${ }^{2}$, P. Barklem ${ }^{3}$, W. Guevara ${ }^{1}$ \\ ${ }^{1}$ Dirección de Astrofísica, Comisión Nacional de Investigación y Desarrollo Aeroespacial - CONIDA, Luis \\ Felipe Villarán 1069, San Isidro, Lima, Perú. \\ 2 The Observatories of the Carnegie Institution for Science; 813 Santa Barbara Street, Pasadena, CA 91101, \\ USA. \\ ${ }^{3}$ Department of Astronomy and Space Physics, Uppsala University, Box 515, 751-20 Uppsala, Sweden
}

\section{RESUMEN}

Este trabajo consiste en determinar la temperatura efectiva de una muestra de 62 estrellas de tipo solar mediante el método de ajuste de perfiles teóricos a la linea espectral Ha (6562.8 A). Las estrellas analizadas han sido observadas usando el Telescopio Harlan J. Smith de $2.7 \mathrm{~m}$ del Observatorio McDonald de la Universidad de Texas mientras que los perfiles teóricos se basan en modelos de atmósferas estelares y teoría cuántica mas recientes. Nuestros resultados poseen un margen de error interno de $\approx 20 \mathrm{~K}$, lo cual nos permite comparar nuestras temperaturas con aquellas determinadas usando otros métodos y especular sobre el origen de las diferencias observadas.

Descriptores: Perfiles de lineas espectrales, atmósferas estelares.

\section{ABSTRACT}

The effective temperature for a sample of 62 solar-type stars has been determined fitting theoretical spectral profiles to observed Ha lines $(6562.8 \mathrm{~A})$. The stars analyzed has been observed using the $2.7 \mathrm{~m}$ Harlan J. Smith telescope at the McDonald Observatory of the University of Texas. The theoretical profiles are based on recent models of stellar atmospheres and quantum theory. The calculated temperatures have internal errors of $\approx 20 \mathrm{~K}$ with other techniques and speculate about the origin of the observed differences.

Keywords: Spectral line profiles, Stellar Atmospheres

\section{INTRODUCCIÓN}

La temperatura efectiva es uno de los parámetros mas importantes de una estrella y se define en función del flujo de energía $(\boldsymbol{F})$, es decir la cantidad de energía emitida en la superficie de una estrella por unidad de tiempo y unidad de área:

$$
\int_{0}^{\infty} F_{\lambda} d \lambda=\sigma T_{\text {eff }}^{4}
$$

Donde: $\sigma$ es la constante de Stefan Boltzmann $\sigma=5.67040 \times 10-5 \mathrm{erg} \mathrm{cm}^{-2} \mathrm{~s}^{-1} \mathrm{k}^{-4}$.
Existen distintos métodos para determinar la temperatura efectiva de una estrella. Los mas utilizados son: el método del flujo infrarrojo (IRFM) $[2,3,10]$, el método de la relación de las profundidades de líneas espectrales (LDR) [5,6], método del equilibrio de excitación de las líneas de $\mathrm{Fe}$ (R09) [11] y el método de las líneas de Balmer [1 ].En el estudio de atmósferas estelares el hidrógeno es una elemento importante ya que es una de las fuentes principales de absorción

continua. En estrellas frías de tipo espectral F, G, $\mathrm{K}$, las alas de las líneas espectrales correspondientes a la serie de Balmer son utilizadas como indicadores de temperatura, 
generalmente asumiendo que se forman en equilibrio termodinámico local (LTE) $[1,4,7]$.

En el óptico, la línea de absorción Ha de la serie de Balmer $(n=2)$ habitualmente es utilizada para estimar la temperatura efectiva debido que la profundidad de sus alas presentan una alta sensibilidad a la temperatura. Nosotros determinamos la temperatura efectiva para una muestra de 62 estrellas de tipo solar mediante el método de las líneas de Balmer, utilizando el perfil de la línea Ha de la serie.

\section{$2 . \quad$ DATOS OBSERVACIONALES Y MODELOS DE PERFILES H $\alpha$ TEORICOS}

Los datos tratados en este trabajo corresponden a una muestra de 62 espectros de estrellas de tipo solar, en la línea espectral Ha. Los espectros observados poseen una alta señal/ruido $(\mathrm{S} / \mathrm{N} \approx$ $200)$ y una alta resolución espectral $(R \approx 60000)$. Estos datos han sido obtenidos con el Telescopio Harlan J. Smith de $2.7 \mathrm{~m}$ en el Observatorio McDonald de la Universidad de Texas, EEUU.

Los modelos de perfiles $\mathrm{Ha}$ teórico han sido calculados a partir de modelos de atmósferas estelares MARCS [8]. Esta malla de modelos fue calculada como en Barklem et al. (2002) y tiene la característica de ser muy fina, lo que nos permitirá obtener temperaturas de alta precisión. La malla de perfiles $\mathrm{Ha}$ teóricos posee las siguientes características: en Temperatura: [5500, 6100] K, en log g: $[4.20,4.65]$ dex y en $[\mathrm{Fe} / \mathrm{H}]:[-0.30,0.30]$ dex, con un espaciamiento de $10 \mathrm{~K}$ en temperatura y 0.05 dex en log g y $[\mathrm{Fe} / \mathrm{H}]$, lo que la hace una de las mallas más finas actualmente en uso.

\section{MEDICION DE LA TEMPERATURA EFECTIVA USANDO PERFILES Ha.}

El método de ajuste de perfiles $\mathrm{Ha}$ consiste en determinar la temperatura efectiva a partir de las alas de la linea espectral. Para analizar estos espectros se cuenta con mallas de modelos de perfiles $\mathrm{Ha}$ y espectros observados. Este análisis consiste en montar un perfil observado con un perfil teórico, con el objetivo de lograr un mejor ajuste entre ambos perfiles (ver la figura 1), previamente se hace una búsqueda minuciosa de regiones limpias sin presencia de líneas telúricas que contaminen el espectro (ver la figura 2). Estas líneas telúricas se encuentran sobre varias bandas espectrales conocidas, por ejemplo en el espectro del sol estas lineas son producto de la absorción del dióxido de carbono, vapor de agua $\mathrm{u}$ oxigeno molecular (O2), presentes en la atmósfera terrestre.

Nosotros utilizamos el método de mínimos cuadrados para encontrar el mejor ajuste al perfil observado como se muestra en la figura 3. El rango de temperaturas obtenidas para nuestra muestra es 5631 - $6018 \mathrm{~K}$.

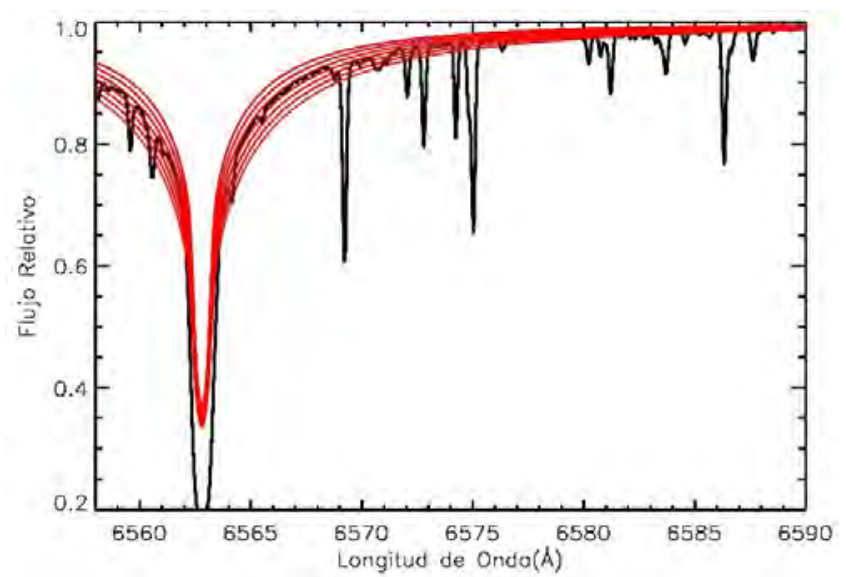

Figura 1. Ajuste del perfil Ha observado para el caso solar superpuesto sobre una malla de perfiles teóricos separados en pasos de $100 \mathrm{~K}$, logg $=4.437$ $\mathrm{y}[\mathrm{Fe} / \mathrm{H}]=0$.

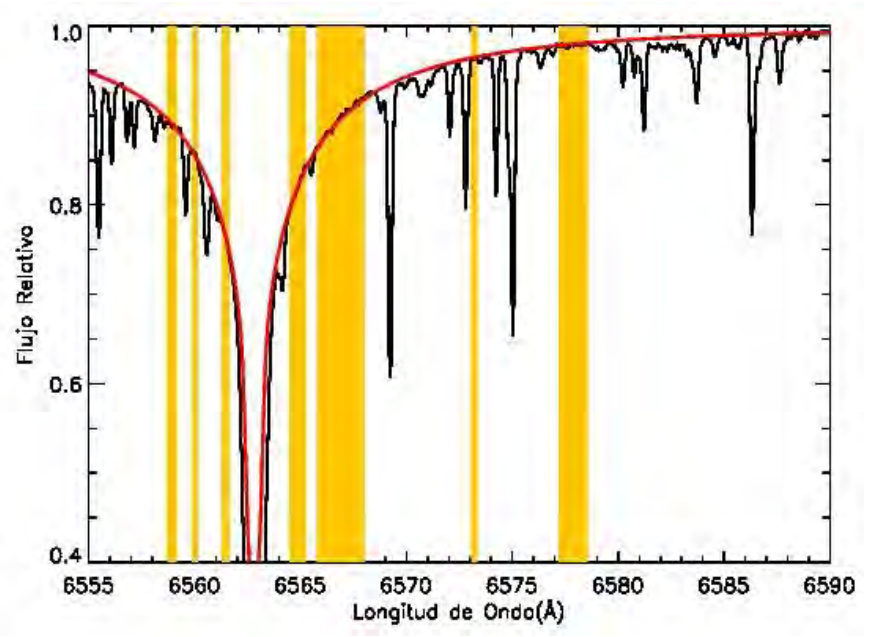

Figura 2. Regiones limpias libres de lineas telúricas para el caso solar. 


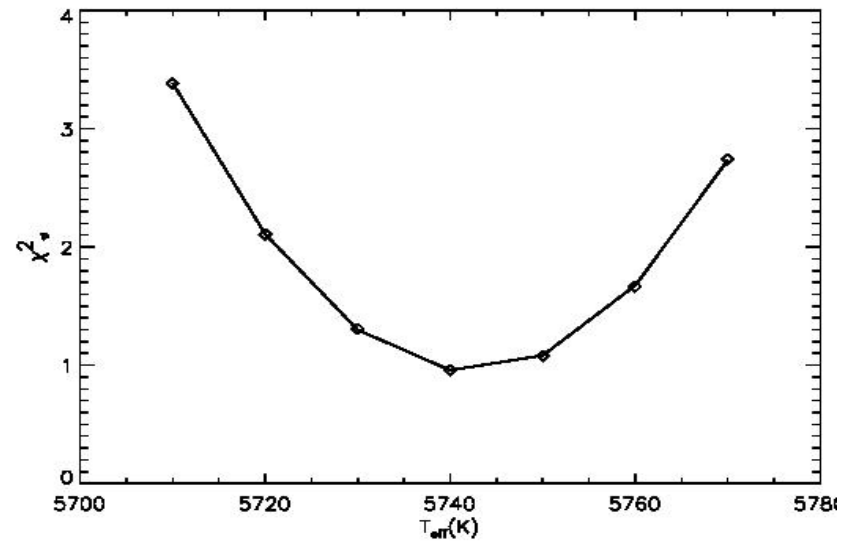

Figura 3. Ajuste de mínimo cuadrados correspondiente a un mejor ajuste del perfil observado para la obtención de la temperatura efectiva para el caso solar.

\section{4.}

\section{RESULTADOS}

La muestra de estrellas fue observada en tres campañas: Abril, Octubre y Noviembre del 2007. $\mathrm{Se}$ obtuvieron temperaturas para todas las estrellas en cada campaña, así como la temperatura solar obtenida a partir de espectros de asteroides. Posteriormente los valores estelares fueron corregidos al punto cero que resulta de igualar a $5777 \mathrm{~K}$ con las temperaturas obtenidas para el Sol, mediante las observaciones indirecta usando los asteroides Ceres y Vesta en cada campaña. Las temperaturas efectivas obtenidas poseen un error aproximadamente de $20 \mathrm{~K}$ y una media obtenida para la temperatura solar aproximadamente de $5752 \mathrm{~K}$. Mediante un análisis diferencial se calculo la temperatura efectiva en la muestra, producto de las correcciones del punto cero para cada campañas, el objetivo de este análisis es reducir los errores sistemáticos presentes en la muestra.

Nuestros resultados de temperatura efectiva ( $T_{\text {eff }}$ (Ha) fueron comparados con las temperaturas efectivas obtenidas mediantes otros métodos ( $\mathrm{Ldr}$, IRFM, $R(09)$ ), ver la figura 4.

En la figura 5, se muestran los residuos (otros métodos - Ha) vs los valores de $\mathrm{T}_{\text {eff }}(\mathrm{H \alpha})$. En este gráfico se puede observar que IRFM (panel intermedio) poseen casi un buen acuerdo con la $\mathrm{T}_{\text {eff }}(\mathrm{H \alpha})$, presentado solo un pequeño desplazamiento que puede ser debido a las diferencias del punto cero. La temperatura efectiva de Ldr (panel superior) muestra un desplazamiento entre las región comprendida $[5550,5700] \mathrm{K}$, que posiblemente se debe a un efecto sistemático.

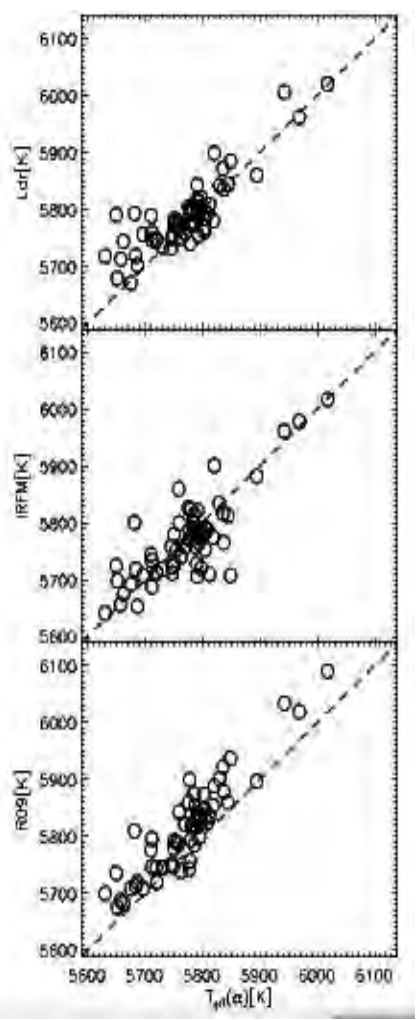

Figura 4. Comparaciones entre la temperatura $L d r$ (panel superior), IRFM (panel intermedio), R09 (imagen inferior) comparados con la temperatura $\mathrm{Ha}$.

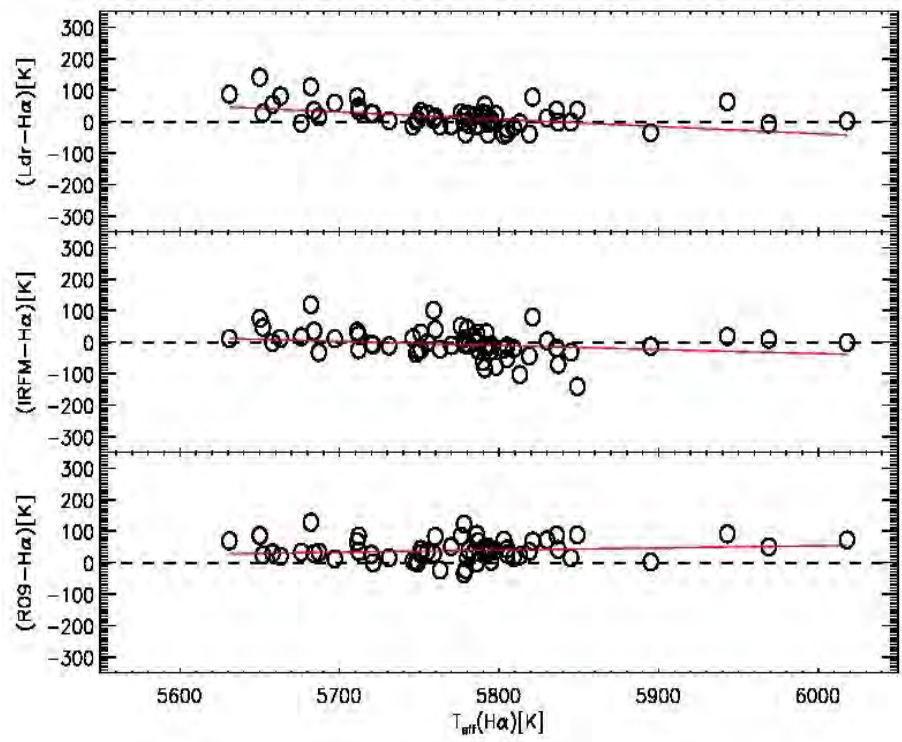


Figura 5. Comparación de los residuos de las temperatura efectiva obtenidas mediante Ldr(panel superior), IRFM(panel intermedio), R09(panel inferior) entre $\operatorname{Teff}(\mathrm{H \alpha})$.

Posteriormente en las figuras 6,7, se muestra las comparaciones de los residuos vs la $\log \mathrm{g}$, $[\mathrm{Fe} / \mathrm{H}]$. En la figura 6 , se muestra que IRFM (panel intermedio) muestra un mejor acuerdo en comparación con los demás métodos y posee una media de -4 y una desviación estándar de 44, mientras que R09 (panel inferior) muestra un desplazamiento casi constante de 46 y una desviación estándar 35. En la figura 7, se observa el residuo Ldr (panel superior) muestra una dependencia en la metalicidad.

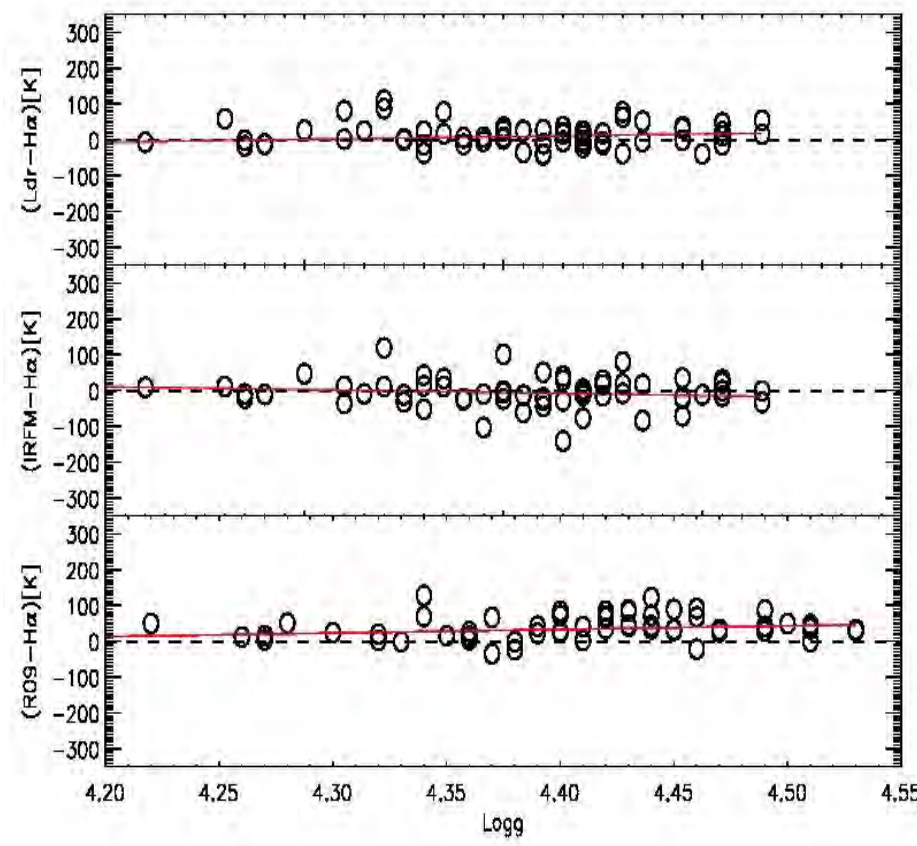

Figura 6. Comparación de los residuos de las temperatura efectiva obtenidas mediante Ldr(panel superior), IRFM(panel intermedio), R09(panel inferior) entre Log $g$.

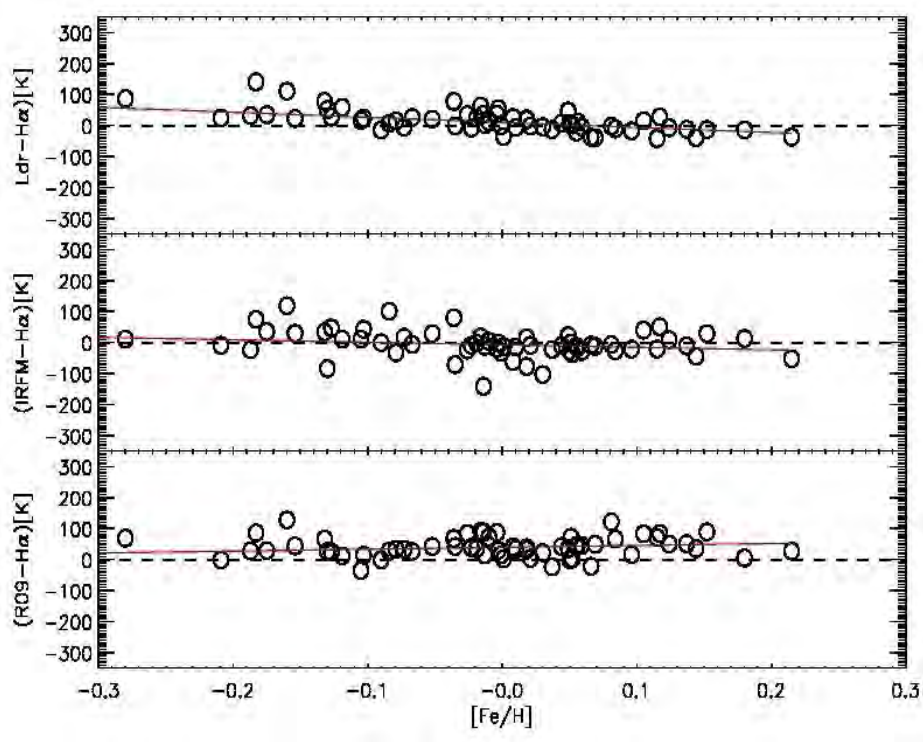

Figura 7. Comparación de los residuos de las temperatura efectiva obtenidas mediante Ldr (panel superior), IRFM(panel intermedio), R09 (panel inferior) entre la $[\mathrm{Fe} / \mathrm{H}]$.

Se analizó las figuras 5 y 7 , con respecto al método de la relación de las profundidades de las líneas espectrales (Ldr), para los casos de metalicidad y temperatura efectiva, mediante un análisis detallado se encontró que este desplazamiento y los puntos dispersados para las regiones donde se encuentran distribuidas las estrellas mas frías de la muestra se deben al efecto de metalicidad. Este método introducido por David F. Gray en los años 80, es sus base no consideraron el parámetro de metalicidad debido que la precisión del calculo de metalicidad estaba en el orden $0.1-0.2$ dex, en la actualidad la precisión de obtener metalicidad esta en los ordenes de $0.03-0.05$ dex, lo que significa que el calculo de temperaturas efectivas obtenidas por la nueva versión de Kovtyukh introduce un pequeño error pero que no es despreciable al calcular la temperatura efectiva, ver las figuras $(8,9,10,11)$. 


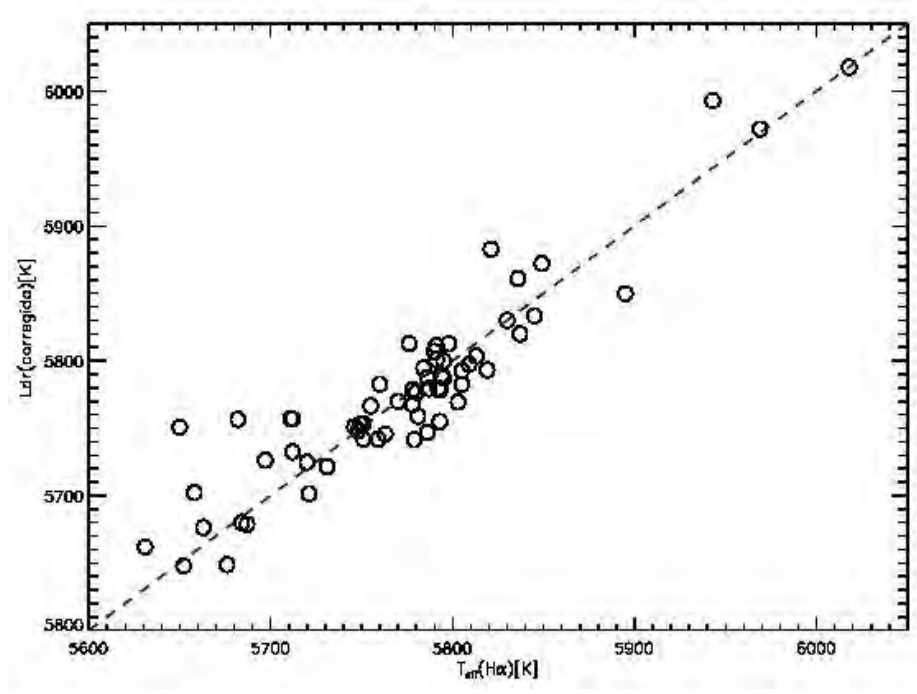

Figura 8. Nueva temperatura efectiva para Ldr.

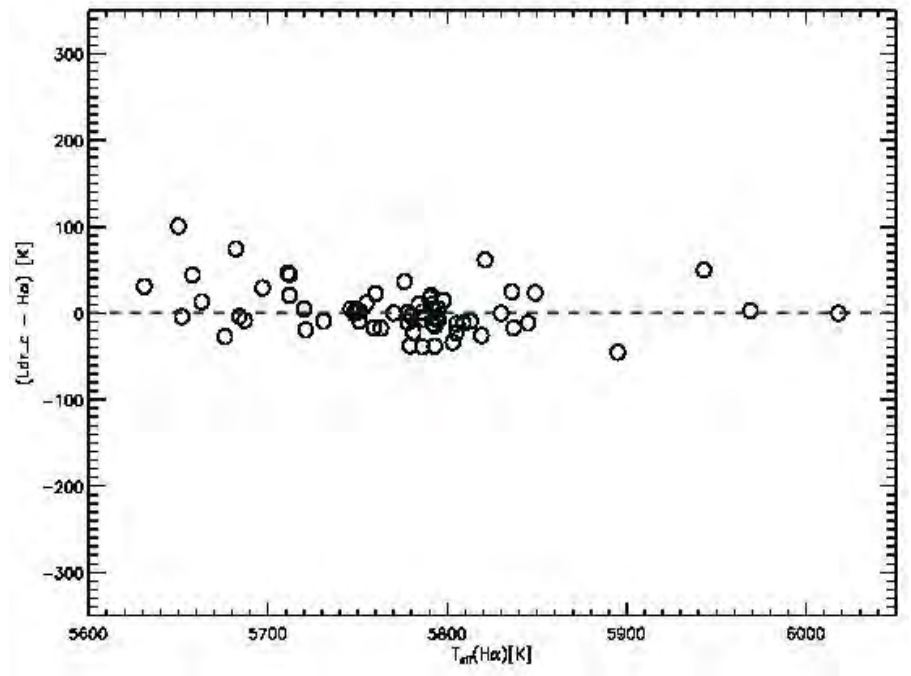

Figura 9. Comparación de los residuos entre $\mathrm{T}_{\text {eff }}(\mathrm{H}$ $\alpha)$.

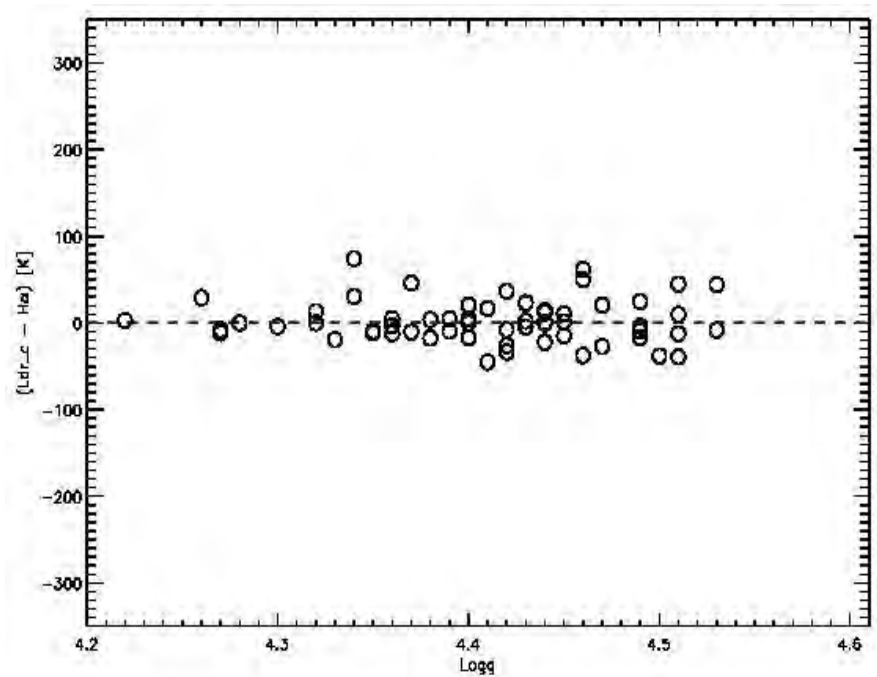

Figura 10. Comparación de los residuos entre Log g.

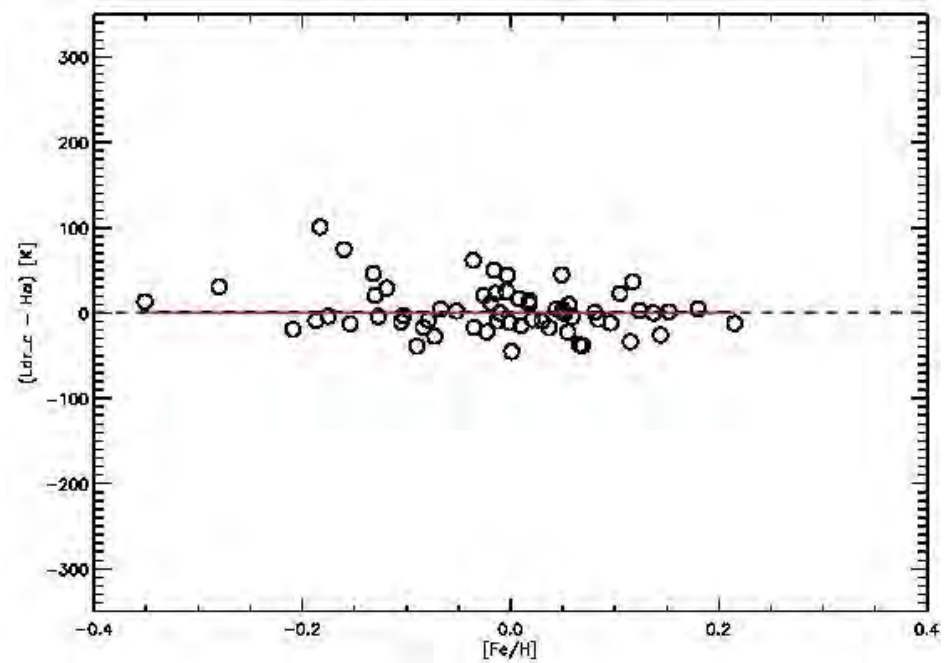

Figura 11. Comparación de los residuos entre $[\mathrm{Fe} / \mathrm{H}]$.

\section{CONCLUSIONES}

Se determinó la temperatura efectiva para 62 estrellas de tipo solar, con un margen de error de aproximadamente $20 \mathrm{~K}$, en comparación a otros métodos que poseen un margen de error de aproximadamente de $50 \mathrm{~K}$. La temperatura efectiva determinada mediante el método de la relación de las profundidades de las líneas espectrales (Ldr), posee una dependencia en metalicidad, es decir que este método debería considerar este parámetro que afecta el calculo de temperatura efectiva como se puedo comprobar en la figura 5 y 7 y se verifico que ese efecto es 
producido por el la $[\mathrm{Fe} / \mathrm{H}]$, como se observo en la figura 11.

\section{AGRADECIMIENTOS}

Agradezco a La CONIDA, Fernando Valle, por su apoyo para la realización de presente trabajo.

\section{REFERENCIAS}

[1] Barklem P.S, Pisnok, O'Mara, Astronomy \& [11] Astrophysics, 385(2002) 951-967.R

[2] Blackwell D., Petford A. and Shallis $M$, Astronomy \& Astrophysics,82(1980) 249-252.

[3] Blackwell D. et al., Astronomy \& Astrophysics, 159(1986)217-222.

[4] Bohm-Vitense E., 1992, "Introduction to stellar astrophysic",vol.2.

[5] Gray D. And Johanson H, Astronomical Society of the Pacific,103(1991) 439-443.
[7] Gray, D. F,1992, " The observation and analysis of stellar photospheres".

[8] Gustafsson et al. , Electrochemical power sources, Peter Peregrinus Ltd. 1980.

[9] Kovtyukh V., Soubiram C., Belik I. And Gorlova N., Astronomy \& Astrophysics, 411(2003)559-564.

[10] Ramírez I., Meléndez J., Astronomy \& Astrophysics, 626(2005)446-464.

Ramirez I., J. Melendez and M. Asplund, Astronomy \& Astrophysics, 508(2009)L17L20.

Email: veronicadce@gmail.com dcornejo@conida

[6] Gray D., Astronomical Society of the Pacific, 106(1994)1248-1257. 